\title{
Correction: Non-invasive diagnosis of retinoblastoma using cell-free DNA from aqueous humour
}

Gerrish A, Stone E, Clokie S, et al. Non-invasive diagnosis of retinoblastoma using cell-free DNA from aqueous humour (Br J Ophthalmol 2019;103:721-4).

The authors have noticed that because of a sample categorisation error prior to sequencing, the RB1 mutations reported for two samples in Table 1 are incorrect. This error also affected some of the data in the paragraph under the heading 'Variant detection in cfDNA from patients undergoing IVC.' This is the corrected paragraph: As a proof of principle, we sequenced cfDNA from two IVC samples. Diagnostic testing had previously identified a germline mutation in patient 1 but the remaining somatic variant in patient 1 and the two somatic variants in patient 2 were unknown due to lack of tDNA. The germline mutation was detected in both the gDNA and cfDNA samples of patient 1 . In addition, three somatic variants were identified in the AH cfDNA samples (table 1). These were complete $\mathrm{LOH}$ of chromosome 13 in patient 1 plus SNV c.1078dupA p.(Ser360Lysfs*2) and a region of LOH (chr 13; 40,803,985-115,064,542) in patient 2 . The mutation allele frequency of these variants showed a similar pattern to the enucleated cfDNA samples, suggesting that the majority of the cfDNA in IVC samples is also derived from the tumour.

\begin{tabular}{|c|c|c|c|c|c|}
\hline & & & gDNA & tDNA & cfDNA \\
\hline Patient & (ng/ul) & RB1 mutation & $\%$ mutation & Result / \% mutation & Result / \% mutation \\
\hline \multirow[t]{2}{*}{ E1 } & 2.12 & c. $1363 C>\operatorname{Tp} .\left(\operatorname{Arg} 455^{*}\right)$ & & 76 & 91 \\
\hline & & $\mathrm{LOH}$ & & Complete LOH & Complete LOH \\
\hline \multirow[t]{2}{*}{ E2 } & 228 & c. $751 C>\operatorname{Tp} .\left(\operatorname{Arg} 251^{*}\right)$ & & 91 & 99 \\
\hline & & $\mathrm{LOH}$ & & Complete LOH & Complete LOH \\
\hline \multirow[t]{2}{*}{ E3 } & 0.183 & c.1959dupA & & 76 & 87 \\
\hline & & $\mathrm{LOH}$ & & Partial LOH & Partial LOH \\
\hline \multirow[t]{2}{*}{ E4 } & 394 & c. $763 C>\operatorname{Tp} .\left(\operatorname{Arg} 255^{*}\right)$ & & 99 & 90 \\
\hline & & $\mathrm{LOH}$ & & Partial LOH & Partial LOH \\
\hline \multirow[t]{2}{*}{ E5 } & 0.169 & c.1251_1252delAA & & 91 & 94 \\
\hline & & $\mathrm{LOH}$ & & Partial LOH & Partial LOH \\
\hline \multirow[t]{2}{*}{ E6 } & 0.141 & Deletion exons 1-17 & & Deletion exons 1-17 & Deletion exons 2-17 \\
\hline & & Deletion exons $25-27$ & & Deletion exons $25-27$ & Deletion exons $24-27$ \\
\hline \multirow[t]{2}{*}{ E7 } & 244 & c.1496_97dup p.Arg500Alafs $² 0$ & 8 & 90 & 94 \\
\hline & & $\mathrm{LOH}$ & & Partial LOH & Partial LOH \\
\hline \multirow[t]{2}{*}{ E8 } & 1.96 & c.1072 C>Tp. $\left(\operatorname{Arg} 358^{*}\right)$ & $21+$ & 97 & 100 \\
\hline & & $\mathrm{LOH}$ & & Partial LOH & Partial LOH \\
\hline \multirow[t]{2}{*}{ E9 } & 1.01 & c. $958 \mathrm{C}>\operatorname{Tp} .\left(\operatorname{Arg} 320^{*}\right)$ & & 43 & 44 \\
\hline & & c. 1981 C >Tp. $\left(\operatorname{Arg} 661^{*}\right)$ & & 46 & 46 \\
\hline \multirow[t]{2}{*}{ E10 } & 1.38 & c.147delT & & 38 & 46 \\
\hline & & c. $1330 \mathrm{C}>\mathrm{Tp} .\left(\mathrm{G} \ln 444^{*}\right)$ & & 50 & 44 \\
\hline \multirow[t]{2}{*}{ IVC 1} & $<0.100$ & $\mathrm{LOH} \dagger$ & & N/A & Complete LOH \\
\hline & & c. $1666 C>\operatorname{Tp} .\left(\operatorname{Arg} 556^{*}\right)$ & 39 & & 100 \\
\hline \multirow[t]{2}{*}{ IVC 2} & $<0.100$ & $\mathrm{LOH} \dagger$ & & N/A & Partial LOH \\
\hline & & c. 1078dupA p.(Ser360Lysfs*2) $†$ & & & 80 \\
\hline
\end{tabular}

Results are shown as percentage of mutation sequencing reads in GDNA, tDNA and cfDNA where appropriate. Complete LOH corresponds to LOH of whole chromosome 13; partial LOH indicates LOH of parts of chromosome 13, encompassing $13 q 14$. tPreviously undetected.

$\mathrm{AH}$, aqueous humour; IVC, intravitreal chemotherapy; LOH, loss of heterozygosity; MPS, massively parallel sequencing; RB, retinoblastoma; cfDNA, cellfree DNA; gDNA, genomic DNA; tDNA, tumour DNA. 
Open access This is an open access article distributed in accordance with the Creative Commons Attribution 4.0 Unported (CC BY 4.0) license, which permits others to copy, redistribute, remix, transform and build upon this work for any purpose, provided the original work is properly cited, a link to the licence is given, and indication of whether changes were made. See: https://creativecommons.org/licenses/by/4.0/.

(C) Author(s) (or their employer(s)) 2020. Re-use permitted under CC BY. Published by BMJ.

Br J Ophthalmol 2019;104:415-416. doi:10.1136/bjophthalmol-2018-313005corr1

Q Check for updates 